\title{
Analysis of Mode Behavior in a Waveguide with Graded Index and Gain
}

\author{
SEIJI MUKAI AND HIROYOSHI YAJIMA, MEMBER, IEEE
}

\begin{abstract}
Numerical analysis has been carried out for a waveguide with graded index and gain. It is shown that the radiation peak of the fundamental mode is deflected as the gradient of either the index or gain increases. The high-order modes also change their peak intensity ratio as the gradient of index or gain increases. The behavior of the fundamental mode is suitable for use in a beam scanner while that of higher-order modes is suitable for use in a deflection switching of laser beams. The analysis also shows that the modal gain of higher-order modes becomes larger than that of the fundamental mode as the gradient of the index and gain increases, which provides some explanation for the wide laser beam scanning observed by Scifres et al.
\end{abstract}

\section{INTRODUCTION}

$\mathrm{C}$ ONTROLLED deflection and switching of laser beams are important for many applications. Beam deflection and switching are currently performed by optical-mechanical and bulk electrooptic or acoustooptic systems. It is desirable for higher reliability and miniaturization of the system to deflect or switch light without using such macroscopic optical systems. Scifres $e t$ al. showed that a beam from a gain-guided semiconductor laser was deflected by controlling the injection current into twin stripes [1], [2]. Shore and Hartnett, and Katz explained that the beam deflection was caused by the tilting of the phase plane of fundamental mode [3], [4]. In addition, Chen and Wang reported that the far-field patterns of laser diodes vary when the current distribution is changed for the laser diode of built-in step index type [5]. They explained that the variation of far-field pattern is due to the coupling of higherorder modes into the fundamental mode through mode locking.

In the present paper, we analyze higher-order mode behavior in the waveguide with asymmetric distribution of refractive index and gain, which gives new possibilities of deflection switching of laser beams. In Section II, we describe a flamework for the analysis of a two-dimensional active waveguide. The formulation is similar to the one detailed by Streifer $e t$ al. [6]. In Section III, we solve the wave equation in the waveguides where both refractive index and gain vary linearly with position. The solutions of the equations give some insight into the behavior of the near-field and far-field patterns of asymmetrically pumped lasers. In particular, an explanation based on mode change is presented for the mechanism of experimentally observed beam deflection. In Section IV, we discuss the application of asymmetrically pumped lasers to the spatial modulation devices.

Manuscript received September 26, 1983; revised March 2, 1984.

S. Mukai was with the Optoelectronics Section, Electrotechnical Laboratory, Niiharigun, Ibaraki 305, Japan. He is now with the California Institute of Technology, Pasadena, CA 91125.

H. Yajima is with the Optoelectronics Section, Electrotechnical Laboratory, Niiharigun, Ibaraki 305, Japan.

\section{FRAMEWORK OF ANALYSIS}

In Fig. 1, we show the cross section of a waveguide used to model the active layer of a semiconductor laser. The waveguide is characterized by the following distribution of the complex dielectric constant $\epsilon(x, y)$

$$
\epsilon(x, y)=\epsilon_{a}+\Delta \epsilon(x) ; \quad|y|<d / 2
$$

and

$$
\epsilon(x, y)=\epsilon_{c} ; \quad|y|>d / 2
$$

where $x$ and $y$ indicate the position along the direction parallel to the perpendicular to the junction plane, respectively. Here, $d$ is the active region thickness. The function $\Delta \epsilon(x)$ is assumed to take nonzero values for $x_{1}<x<x_{2}$ and to be zero for $x<$ $x_{1}$ and $x_{2}<x$. We restrict our analysis to the mode with transverse electric field because such modes usually dominate in semiconductor lasers.

Assuming a time dependence $\exp (i \omega t)$ and $z$ dependence $\exp (-i \beta z)$, we find from Maxwell's equation that $E_{x}$, the $x$ component of the electric field, satisfies the equation

$$
\frac{\partial}{\partial x}\left(\frac{E x}{\epsilon} \frac{\partial \epsilon}{\partial x}\right)+\left(\frac{\partial^{2}}{\partial x^{2}}+\frac{\partial^{2}}{\partial y^{2}}\right) E_{x}-\left(\beta^{2}-\epsilon K_{0}^{2}\right) E_{x}=0
$$

where $\beta$ is a propagation constant, $K_{0}=2 \pi / \lambda_{0}$, and $\lambda_{0}$ is the free space wavelength. Following a conventional procedure [6], [7], we seek a solution of product form to separate variables.

$$
E_{x}(x, y)=f(x) g(y) .
$$

This approximation is based on the assumption that the variation of $\Delta \epsilon(x)$ is small compared to the difference $\epsilon_{a}-\epsilon_{c}$ so that $g(y)$ is approximately independent of $x$. Then, $f(x)$ satisfies the following equation:

$$
\frac{d^{2} f}{d x^{2}}-\left[\beta^{2}-\left(\beta_{m}^{2}+K_{0}^{2} \Gamma \Delta \epsilon\right)\right] f=0
$$

where $\Gamma$ is a filling factor [6].

$$
\Gamma=\int_{-d / 2}^{d / 2}|g(y)|^{2} d y / \int_{-\infty}^{\infty}|g(y)|^{2} d y
$$

and $\beta_{m}$ and $g(y)$ are given by

$$
\frac{d^{2}}{d y^{2}} g(y)-\left[\beta_{m}^{2}-\epsilon^{\prime}(y) K_{0}^{2}\right] g(y)=0
$$




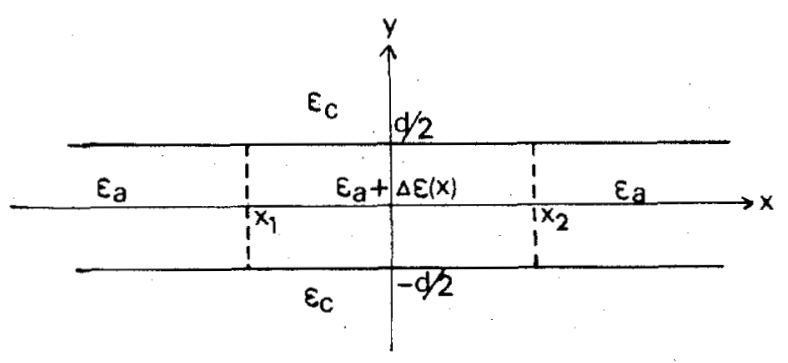

Fig. 1. Cross section of the waveguide geometry.

where

$$
\begin{array}{lll}
\epsilon^{\prime}(y)=\epsilon_{a} & \text { for } & |y|<d / 2 \\
\epsilon^{\prime}(y)=\epsilon_{c} & \text { for } & |y|>d / 2 .
\end{array}
$$

Note $g(y)$ and $\beta_{m}$ are first obtained from (7) for the fundamental mode in the $y$ direction. Using the value of $\beta_{m}$ together with $\Gamma$ obtained from $(6), f(x)$ and $\beta$ can then be obtained by solving (5) using a combination of Runge-Kutta's method [8] and Newton-Raphson's method [8]. The gain of the guidedwave is given as two times of the imaginary part of $\beta$. The detail of the computation procedure is described in the Appendix.

The far-field pattern $I(\theta)$ was calculated according to the relation

$$
I(\theta)=\cos ^{2} \theta\left|\int_{-\infty}^{\infty} f(x) \exp \left(i K_{0} x \sin \theta\right) d x\right|^{2}
$$

where $\theta$ is an angle measured from the axis of the waveguide to the direction of $x$, and its sign is such that the positive $\theta$ lies on the side of positive $x$.

\section{Numerical COMPUTATIONS}

In the analysis, we assume the waveguide parameters as follows; $d=0.22 \mu \mathrm{m},\left(x_{1}, x_{2}\right)=(-5 \mu \mathrm{m}, 5 \mu \mathrm{m}), \epsilon_{a}=13.1$ $0.023 i$ and $\epsilon_{c}=11.4$. The free space wavelength of transmitted light $\lambda_{0}$ was taken to be $0.84 \mu \mathrm{m}$. These values of $\epsilon_{a}$ and $\epsilon_{c}$ correspond to $\mathrm{Al}_{0.05} \mathrm{Ga}_{0.95} \mathrm{As}$ and $\mathrm{Al}_{0.35} \mathrm{Ga}_{0.65} \mathrm{As}$ as a material for an active layer and confinement layer [9] , [10] . The filling factor $\Gamma$ of the waveguide was calculated to be 0.69 .

For a simplified expression of asymmetry, the spatial variation of $\epsilon(x)$ was assumed to be linear.

$$
\Delta \epsilon(x)=\left(-a_{1}+a_{2} i\right) x+b
$$

where $a_{1}$ and $a_{2}$ are real constants and $b$ is a complex constant. The variation of $\Delta \epsilon(x)$ can be realized either by introducing asymmetric built-in index structures in the waveguide or by introducing asymmetry in the spatial distribution of injected carriers. In the latter case, $\Delta \epsilon(x)$ was assumed to depend linearly on the carrier density.

$$
\Delta \epsilon(x)=\left(-c_{1}+c_{2} i\right) N(x)
$$

where $c_{1}$ and $c_{2}$ are real constants, and $N(x)$ is the local injected carrier density in the active region which is expressed in units of $10^{18} / \mathrm{cm}^{3}$. The function $N(x)$ was assumed to be given by

$$
N(x)=2.0+A x
$$

where $A$ is the gradient factor and $x$ is the position in microm-
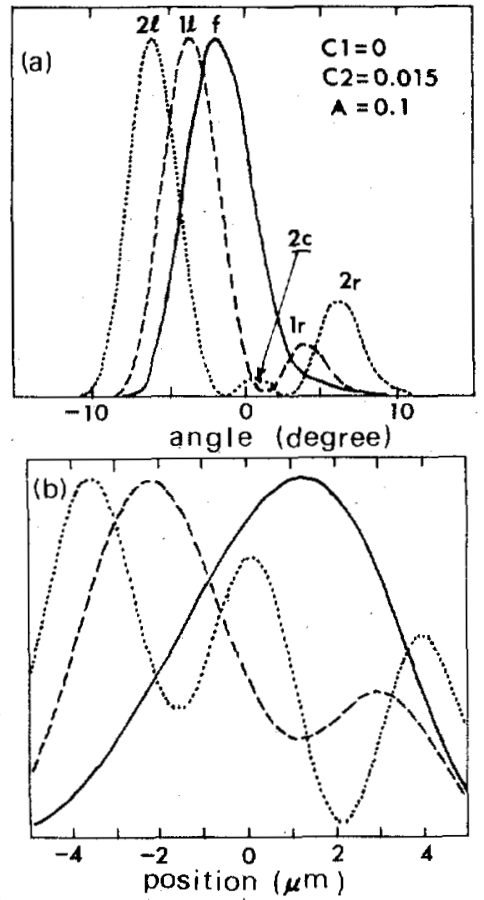

Fig. 2. Far-field patterns (a) and near-field patterns (b) of a waveguide with uniform index and graded gain with parameters $\left(c_{1}, c_{2}, A\right)=$ $(0,0.015,0.1)$. In (a) $f$ indicates the peak of the fundamental mode radiation, $1 l$ and $1 r$ indicate peaks of the first-order modes, and $2 l$, $2 c$, and $2 r$ indicate the peaks of the second-order mode. In (b) the solid line, broken line, and dotted line indicate fundamental, firstorder, and second-order modes, respectively.

eters. The ratio $c_{1} / c_{2}$ deduced from experimental data in actual laser diodes ranges from 0.5 to 4 [11], [12]. In this paper, analyses were performed for the ratio of $0,0.5,1$, and 3.6.

\section{A. Waveguide with Uniform Index and Graded Gain}

First, we investigate the effect of graded gain in the waveguide on the behavior of guided modes. Before proceeding with the discussion, we note that the waveguide of uniform refractive index and gain gives symmetrical far-field and nearfield patterns for every guided mode.

Fig. 2(a) shows far-field patterns of guided modes in a waveguide characterized by $\left(c_{1}, c_{2}, A\right)=(0.0,0.015,0.1)$. The effect of graded gain to the fundamental mode is apparent in the radiation angle; the peak of $f$ shifts to the lower gain side. As for higher-order modes, the change in far-field patterns is more apparent in the peak intensity ratio than the peak shift. The peaks on the higher gain side, $2 r$ and $1 r$, become lower than those on the lower gain side, $2 l$ and $1 l$. Fig. 2(b) shows near-field patterns of guided modes. The peaks of the fundamental mode shift to the side of higher gain. As for higher modes, the peak shift is small but again the peak intensity ratio changes. The peaks on the lower gain side become large, whereas those on the higher gain side become small. The dependence of modal gain $g_{i}$, radiation angle of major peaks, and normalized intensity of minor peaks for each mode on the gradient factor $A$ are shown in Fig. 3. The radiation angle of the fundamental mode shifts to the lower gain side in proportion to $A$, whereas the radiation angle of higher-order modes is insensitive to $A$. The modal gain of the fundamental mode 


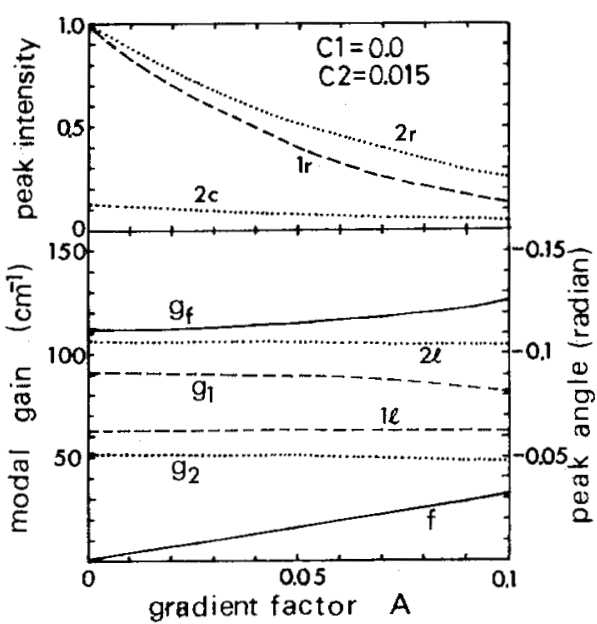

Fig. 3. Modal gain, angle of far-field peaks, and normalized intensity of minor far-field peaks as a function of gradient factor $A . g_{f}, g_{1}$, and $g_{2}$ are modal gains for the fundamental, first-order, and secondorder modes. $f, 1 l$, and $2 l$ are angles of the main peaks corresponding to $f, 1 l$, and $2 r$ in Fig. 2 . $1 r, 2 r$, and $2 c$ are normalized intensities of the peaks corresponding to $1 r, 2 r$, and $2 c$ in Fig. 2 .

increases as $\boldsymbol{A}$ increases. This is because the field distribution of the fundamental mode shift to the higher gain side in the waveguide. Modal gain of higher-order modes decreases slightly as $A$ increases.

\section{B. Waveguide with Graded Index and Gain}

Next, we investigate the case when both $c_{1}$ and $c_{2}$ are nonzero. Fig. 4(a) shows the far-field patterns of guided modes in a waveguide characterized by $\left(c_{1}, c_{2}, A\right)=(0.075,0.015$, 0.1 ). We see from (12) that if $c_{1}$ and $c_{2}$ have the same sign, the gradient of the real and imaginary parts of $\Delta \epsilon$ have opposite signs. This means that the higher gain side of the waveguide has a lower refractive index, and the lower gain side has a higher refractive index. The far-field patterns are similar to those in Fig. 2(a), but their radiation peak angles are relatively larger than those in Fig. 2(a). The radiation peak of the fundamental mode is deflected to the lower gain and higher refractive index side. The higher-order modes change their peak intensity ratios and the peaks on the lower gain side become large when those on the higher gain side become small. Fig. 4(b) shows the near-field patterns of each guided mode. Fundamental, first-, and second-order modes have their main peaks on the lower gain side. It is known generally that, in the waveguide with nonuniform refractive index distribution, the field of the fundamental mode is distributed on the higher index area and those of higher-order modes are distributed on the lower index area. Our computation results indicate that the field distribution of the fundamental mode in the waveguide is more sensitive to the index gradient, whereas those of higher-order modes are more sensitive to the gain gradient.

The dependence of modal gain, radiation angles of major peaks, and normalized intensity of minor peaks for each mode on the gradient factor $\boldsymbol{A}$ is shown in Fig. 5. As $\boldsymbol{A}$ increases, the modal gain of the fundamental mode decreases. This is because the field of the fundamental mode shifts to the lower gain side. The modal gain of the first mode increases as the $A$ increases. The reason seems to be that the minor peak of the

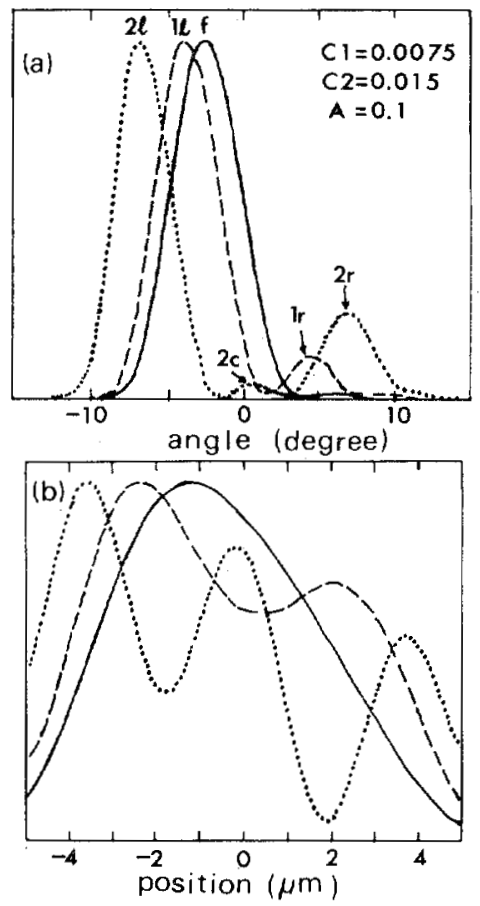

Fig. 4. Far-field patterns (a) and near-field patterns (b) of a waveguide with parameters $\left(c_{1}, c_{2}, A\right)=(0.0075,0.015,0.1)$.

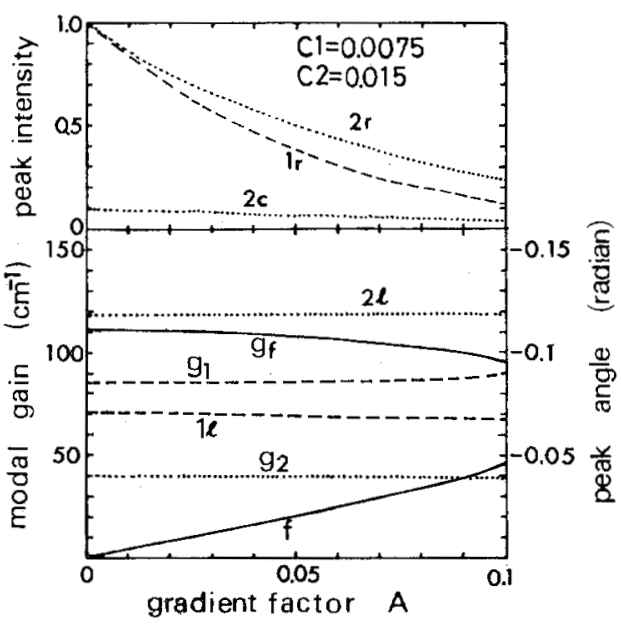

Fig. 5. Modal gain $\left(g_{f}, g_{1}\right.$ and $\left.g_{2}\right)$, angle of far-field peaks $(f, l l$, and $2 l$ ) and normalized intensity of minor far-field peaks $(1 r, 2 r$, and $2 c)$ as a function of gradient factor $A$.

near-field pattern in the higher gain area is not so small as that in Fig. 2(b). As a result, the modal gain of the first-order mode approaches that of the fundamental mode as $A$ increases.

Fig. 6(a) shows the far-field patterns of guided modes in the waveguide characterized by $\left(c_{1}, c_{2}, A\right)=(0.015,0.015,0.1)$ The far-field patterns are similar to those in Fig. 4(a), but their radiation peak angles are larger than those in Fig. 4(a). Fig. 6(b) shows the near-field patterns for each guided mode. Fundamental and second-order modes have their main peaks on the lower gain side. However, the first-order mode has their main peak on the higher gain side. The dependence of modal gain, radiation angles of major peaks, and normalized intensity of minor peaks for each mode on the gradient factor $A$ is shown in Fig. 7. As $A$ is increased, the modal gain of the fundamental mode decreases and that of the first-order mode increases. 

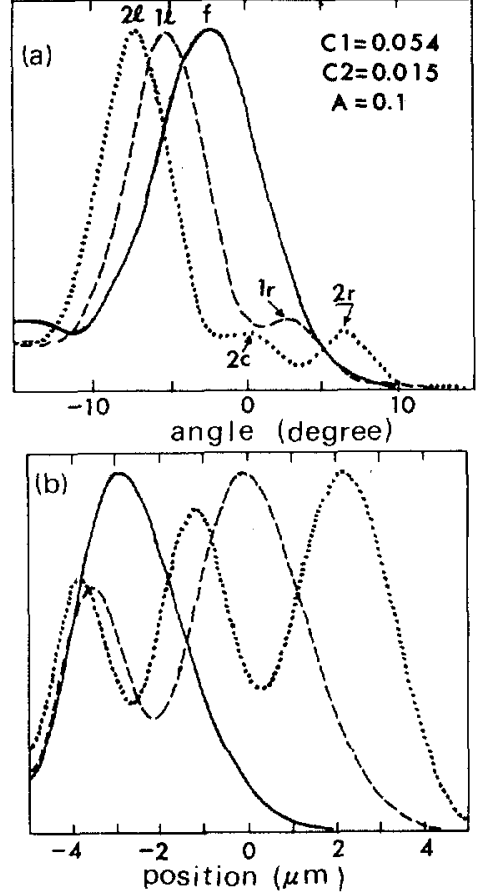

Fig. 6. Far-field patterns (a) and near-field patterns (b) of a waveguide with parameters $\left(c_{1}, c_{2}, A\right)=(0.015,0.015,0.1)$.

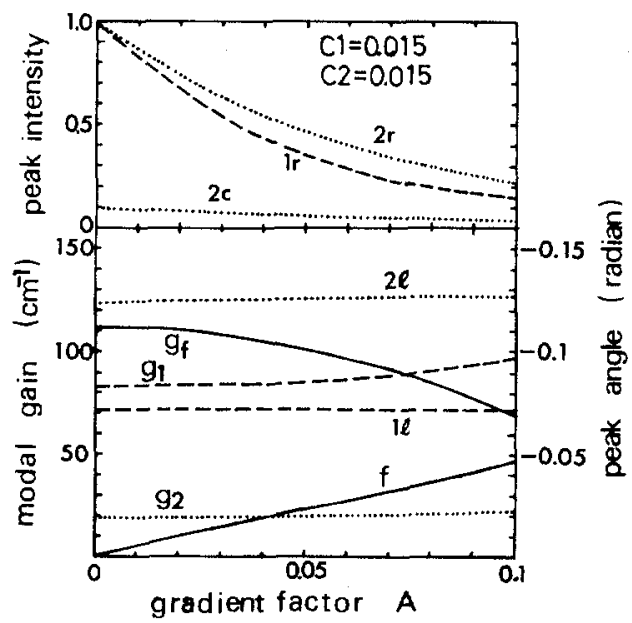

Fig. 7. Modal gain $\left(g_{f}, g_{1}\right.$, and $\left.g_{2}\right)$, angle of far-field peaks $(f, 1 l$, and $2 l$ ), and normalized intensity of minor far-field peaks $(1 r, 2 r$, and $2 c)$ as a function of gradient factor $\boldsymbol{A}$.

The modal gain of the first-order mode becomes larger than that of the fundamental mode for $A>0.074$. The modal gain of the second-order mode increases slightly as $A$ is increased.

Fig. 8(a) shows the far-field patterns of guided-modes in the waveguide characterized by $\left(c_{1}, c_{2}, A\right)=(0.054,0.015,0.1)$. The peak angle of the fundamental and second-order modes are almost the same as those in Fig. 6(b). The radiation peak angle of the first-order mode is larger than that in Fig. 6(b). Radiation around an angle of $-15^{\circ}$ is enhanced for all modes, which reflects the antiguiding property of the guide resulting from the real part of $b$ being a large negative value. Fig. 8(b) shows the near-field pattern of each guided mode. The fundamental mode is localized on the higher refractive index side. The first-order mode also shifts to the higher refractive index side. The second-order mode has its major peak on the higher gain side. The dependence of modal gain, radiation angles of
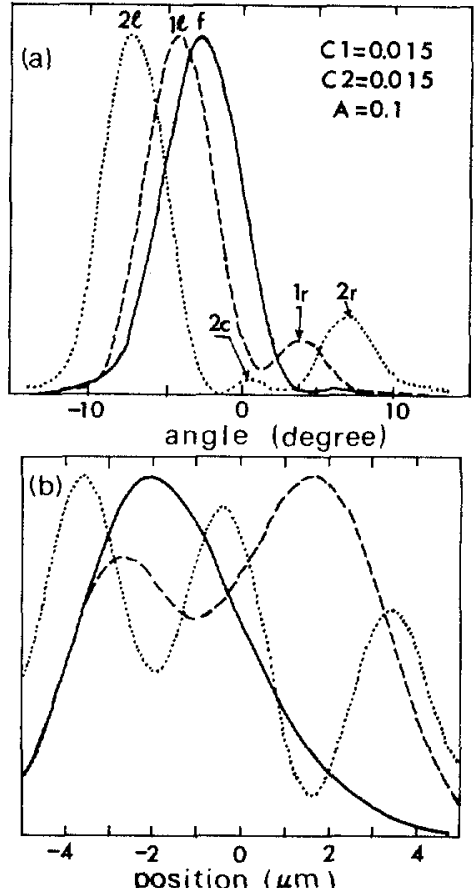

Fig. 8. Far-field patterns (a) and near-field patterns (b) of a waveguide with parameters $\left(c_{1}, c_{2}, A\right)=(0.054,0.015,0.1)$.

major peaks, and normalized intensity of minor peaks on the gradient factor $A$ is shown in Fig. 9. As $A$ increases, the modal gain of the fundamental mode monotonically decreases, while that of the first-order mode increases for $A<0.04$ and decreases for $A>0.04$. The gain of the second-order mode increases monotonically as $A$ increases. As a result, the mode with the highest gain is the fundamental mode for $A<0.034$, the firstorder mode for $0.034<A<0.090$ and the second-order mode for $0.090<A$

In laser diodes, the first lasing mode is the mode with the highest gain. The result in Fig. 9 shows that as $A$ increases, the fundamental mode first lases and modes of higher-order lases afterward. The far-field patterns of the fundamental mode for $A=0.03$, the first-order mode for $A=0.06$, and the secondorder mode for $A=0.1$ are shown in Fig. 9. Scifres et al. state that the beam deflection by an angle as large as $14^{\circ}$ from the normal of the facet was realized by the modification of injected carrier distribution [1]. The present analysis indicates that an expected angle of deflection for the fundamental mode is much smaller than $14^{\circ}$. It is the change of an oscillating mode from the fundamental mode to the higher-order mode that provides such a large angle deflection in the present model.

\section{Summary AND Conclusion}

The features of far-field patterns of guided modes in the waveguide with graded index and gain are summarized as follows.

1) The deflection angle of the fundamental mode increases as the gradient factor increases. The maximum deflection angle is around $5-6^{\circ}$.

2) The far-field pattern of higher-order modes has two main peaks and the intensity ratio of the peaks changes as the gradient factor increases. The peak position is insensitive to the gradient factor. 


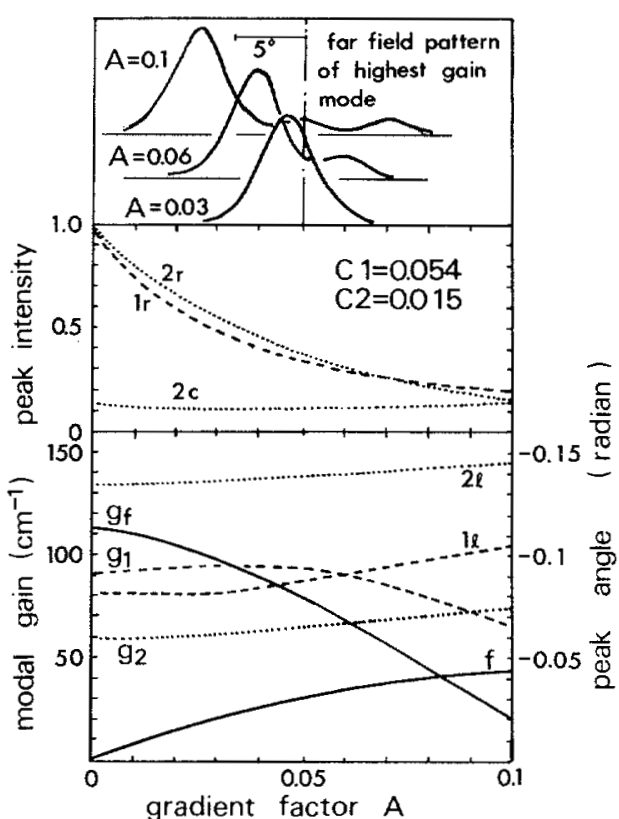

Fig. 9. Modal gain $\left(g_{f}, g_{1}\right.$, and $\left.g_{2}\right)$, angle of far-field peaks $(f, 1 l$, and $2 l)$, and normalized intensity of minor far-field peaks $(1 r, 2 r$, and $2 c)$ as a function of gradient factor $A$. On the top of the figure are also shown far-field patterns of the mode with the highest modal gain at several values of $A$.

3) The deflection angle of peaks increases as the mode number increases.

4) As the gradient factor increases, the gain factor of the fundamental mode decreases, but those of higher-order modes increases. And the gain factor of higher-order modes exceeds that of the fundamental mode for the value of large gradient factor. This means that the mode-jump from lower-order modes to higher-order modes occurs as the gradient factor increases, which gives some explanation of the wide deflection angle of the beam from the twin-stripe laser diode observed by Scifres et al. [1].

We see that the selective transverse-mode oscillation of laser diode gives the possibility of different spatial beam modulation technology by controlling the injection current distribution in the active layer. That is, the fundamental mode excitation is suitable for the beam scanning because the deflection angle of the beam is proportional to the gradient factor. On the other hand, the higher-order mode oscillation is suitable for the deflection switching of the beam because the peak intensity ratio changes with the fixed peak angle as the gradient factor changes. The selective higher-order mode oscillation of the twin-stripe laser diode has been observed by authors [12]. The direct spatial modulation techniques of laser diodes means the functional integration of light sources, and it will provide many applications in optical information processing.

\section{APPENDIX}

Equation (6) was solved numerically for $\beta$ and $f(x)$ with the boundary conditions

$$
f(x) \rightarrow 0 \text { for } x \rightarrow-\infty
$$

and

$$
f(x) \rightarrow 0 \quad \text { for } x \rightarrow+\infty
$$

Since $\Delta \epsilon(x)=0$ for $x \leqslant x_{1}$ or $x \geqslant x_{2}, f(x)$ is of the form

$$
\begin{array}{ll}
f(x)=A \exp (\alpha x)+B \exp (-\alpha x) & \text { for } x \leqslant x_{1} \\
f(x)=C \exp (\alpha x)+D \exp (-\alpha x) & \text { for } x_{2} \leqslant x
\end{array}
$$

where $\alpha=\sqrt{\beta^{2}-\beta_{m}^{2}}$, and $A, B, C$, and $D$ are complex constants. If $\operatorname{Re}(\alpha)>0$, the conditions of (A-1) and (A-2) are equivalent to the following conditions:

$$
B=0
$$

and

$$
C=0 \text {. }
$$

For $x=x_{1}, f(x)$ is normalized to unity and the derivative of $f(x)$ is then $\alpha$ from (A-3) and (A-5). We obtain $f(x)$ for $x_{1}<x<x_{2}$ by integrating (6) by Runge-Kutta's method with initial values $\left[f\left(x_{1}\right),(d / d x) f\left(x_{1}\right)\right]=(1, \alpha)$. Let us assume

$$
f(x)=F\left(x, x_{1}, \alpha\right) \text { for } x_{1}<x<x_{2}
$$

and

$$
\frac{d}{d x} f(x)=G\left(x, x_{1}, \alpha\right) \quad \text { for } \quad x_{1}<x<x_{2} .
$$

Using (A-4) and (A-6) we obtain the following equation for $\beta$ :

$$
F\left(x_{2}, x_{1}, \alpha(\beta)\right)+\frac{1}{\alpha(\beta)} G\left(x_{2}, x_{1}, \alpha(\beta)\right)=0
$$

For a simple description, we represent the left side of (A-10) by $K(\beta)$, and rewrite $(\mathrm{A}-10)$ as

$$
K(\beta)=0 \text {. }
$$

The equation (A-11) is solved by Newton-Raphson's method with a trial first value of $\beta$. The derivative of $K(\beta)$ that is needed to apply Newton-Raphson's method is obtained by the relation

$$
\frac{d}{d \beta} K(\beta)=[K(\beta+\Delta)-K(\beta)] / \Delta
$$

where $\Delta$ is a small value which we put as $10^{-10} / \mu \mathrm{m}$. In the process of finding $\beta, f(x)$ is simultaneously obtained by (A-7). Various solutions of $f(x)$ and $\beta$ corresponding to various modes are obtained by changing the first trial value of $\beta$.

\section{ACKNOWLEDGMENT}

The authors are grateful to S. Ishihara, K. Kikuchi, K. Suzuki, Y. Mitsuhashi, and J. Shimada of Electrotechnical Laboratory, S. Uekusa of Meiji University, and A. Sone of Hamamatsu Photonics Company for their discussion and encouragement.

\section{REFERENCES}

[1] D. R. Scifres, W. Streifer, and R. D. Burnham, "Beam Scanning with twin stripe injection lasers," Appl. Phys. Lett., vol. 33, pp. 702-704, Oct. 1978.

[2] W. Streifer, R. D. Burnham, and D. R. Scifres, "Symmetrical and asymmetrical waveguide in very narrow conducting stripe lasers," IEEE J. Quantum Electron., vol. QE-15, pp. 136-141, Mar. 1979.

[3] K. A. Shore and P. J. Hartnett, "Diffusion and waveguide effects in twin-stripe injection lasers," Opt. Quantum Electron., vol. 14, pp. 169-176, 1982. 
[4] J. Katz, "Electronic beam steering of semiconductor injection lasers," Appl. Opt., vol. 22, pp. 313-317, 1983.

[5] C. Y. Chen and S. Wang, "Effects of the current distribution on the characteristics of the semiconductor laser with a channeledsubstrate planar structure," J. Appl. Phys., vol. 52, pp. 614620, Feb. 1981.

[6] W. S. Streifer, D. R. Scifres, and R. D. Burnham, "Analysis of gain-induced waveguiding in stripe-geometry diode lasers," IEEE J. Quantum Electron., vol. QE-14, pp. 418-428, June 1978.

[7] T. L. Paoli, "Waveguide in a stripe-geometry junction laser," IEEE J. Quantum Electron., vol. QE-13, pp. 662-668, Aug. 1977.

[8] C. E. Fröberg, Introduction to Numerical Analysis, 2nd ed. Reading, MA: Addison-Wesley, 1970.

[9] D. D. Sell, H. C. Casey, Jr., and K. W. Wecht, "Concentration dependence of the refractive index for $\mathrm{n}$ - and p-type GaAs between 1.2 and $1.8 \mathrm{eV}, " J$. Appl. Phys., vol. 45, p. 2650, June 1974.

[10] H. C. Casey, Jr. and M. B. Panish, Heterostructure Lasers. New York: Academic, 1978, ch. 2

[11] D. D. Cook and F. R. Nash, "Gain-induced guiding and astigmatic output beam of GaAs lasers," J. Appl. Phys., vol. 46, pp. 16601672, Apr. 1975.

[12] P. A. Kirkby, A. R. Goodwin, G. H. B. Thompson, and P. R. Selway, "Observation of self-focusing in stripe geometry semiconductor lasers and the development of a comprehensive model of their operation," IEEE J. Quantum Electron., vol. QE-13, pp. 705-719, Aug. 1977.

[13] S. Mukai, H. Yajima, S. Uekusa, and A. Sone, "Transverse secondorder mode oscillations in a twin-stripe laser with asymmetric injection currents," Appl. Phys. Lett., vol. 43, pp. 432-434, Sept. 1983.

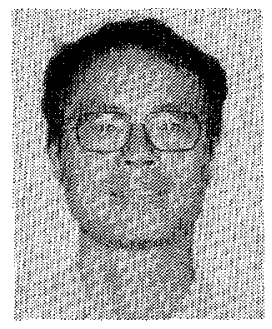

Seiji Mukai was born in Osaka, Japan, in March 1949. He received the B.S., M.S., and Ph.D. degrees in physics from Kyoto University, Kyoto, Japan in 1971, 1973, and 1983, respectively.

In 1973 he joined the Electrotechnical Laboratory, Ibaraki, Japan, where he has been involved in developing semiconductor lasers. He also developed a growth method of quarternary semiconductors for visible emission devices. Since 1983 he has been working as a Visiting Associate with the California Institute of Technology, Pasadena, CA, investigating functional semiconductor lasers.

Dr. Mukai is a member of the Physical Society of Japan and the Japanese Society of Applied Physics.

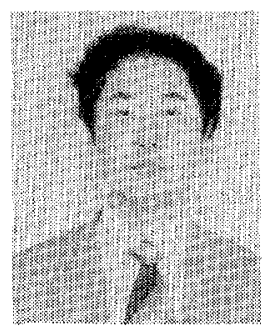

Hiroyoshi Yajima (S'68-M'69) was born in Tokyo, Japan, on August 9, 1941. He received the M.E. and Ph.D. degrees from Keio University, Tokyo, Japan, in 1966 and 1969, respectively.

In 1969, he joined the staff of the Electrotechnical Laboratory, Ibaraki, Japan, From 1976 to 1977 he was a Postdoctoral Fellow at the University of California, San Diego. He is now a Senior Research Scientist at the Electrotechnical Laboratory. He is currently engaged in work on integrated optics.

Dr. Yajima is a member of the Institute of Electronics and Communication Engineers of Japan and the Japan Society of Applied Physics.

\title{
Stabilization of Single Frequency Operation of Coupled-Cavity Lasers
}

\author{
CHARLES H. HENRY AND R. F. KAZARINOV
}

\begin{abstract}
We present a theoretical analysis of cleaved coupled-cavity lasers. Mode selectivity arises from two mechanisms. The first is diffraction loss in the gap between the cleaved sections. The second is based on one section acting as a resonant reflector. Our analysis includes the change of refractive index with carrier density which shifts the cavity resonances and causes mode switching. Above threshold the gains $g_{1}$ and $g_{2}$ of the two cavities are not pinned, but are related for each mode in the form of a curve in the $\left(g_{1}, g_{2}\right)$ plane. The separation of mode curves along a $45^{\circ}$ line determines the ratio of mode intensities. Single mode operation above threshold is described by zones in the $\left(C_{1}, C_{2}\right)$ plane where $C_{1}$ and $C_{2}$ are the currents driving the two cavities. Cases of stable, unstable, and bistable operation are discussed.
\end{abstract}

\section{INTRODUCTION}

$\mathbf{R}$ ECENTLY, there has been intensive experimental and theoretical work on coupled-cavity semiconductor lasers [1]-[12] culminating in a demonstration of error-free transmission over a distance of $119 \mathrm{~km}$ at $420 \mathrm{mbits} / \mathrm{s}$ without re-

Manuscript received October 7, 1983; revised March 2, 1984.

The authors are with AT\&T Bell Laboratories, Murray Hill, NJ 07974. peaters [13]. This achievement required a laser maintaining single frequency operation with the nonlasing modes greatly suppressed during pulsed operation; otherwise excessive errors due to mode partition noise are encountered. The most straightforward way to achieve single frequency operation is by use of a distributed feedback laser [14], [15]. The operation of this laser is simple, but the distributed feedback laser is difficult to fabricate.

In the $119 \mathrm{~km}$ experiment a cleaved coupled-cavity laser previously developed by Tsang et al. [7] - [9] was used [13] . These lasers show impressive mode selectivity (high ratio of lasing to nonlasing mode intensities). In contrast to the DFB lasers, cleaved coupled-cavity lasers are relatively easy to fabricate, but are more complex to operate. They tend to change modes during pulsed operation and only remain on one mode over a limited range of current. Use of coupled-cavity lasers in optical communication systems must be preceded by a precise understanding of how coupled-cavity lasers work, both at threshold and above threshold, and procedures for high power single 